\title{
Perfusion Imaging to Select Patients with Large Ischemic Core for Mechanical Thrombectomy
}

\author{
Basile Kerleroux, ${ }^{a}$ Kevin Janot, ${ }^{\text {a }}$ Cyril Dargazanli, ${ }^{b}$ Dimitri Daly-Eraya, ${ }^{b}$ Wagih Ben-Hassen, ${ }^{c}$ \\ François Zhu, ${ }^{\mathrm{d}}$ Benjamin Gory, ${ }^{\mathrm{d}}$ Jean François Hak, ${ }^{\mathrm{e}}$ Charline Perot, ${ }^{\mathrm{f}}$ Lili Detraz, ${ }^{\mathrm{g}}$ Romain Bourcier, ${ }^{\mathrm{g}}$ \\ Aymeric Rouchaud, ${ }^{\mathrm{h}}$ Géraud Forestier, ${ }^{\mathrm{h}}$ Joseph Benzakoun, ${ }^{\mathrm{c}}$ Gaultier Marnat, ${ }^{\mathrm{i}}$ Florent Gariel, ${ }^{\mathrm{i}}$ \\ Pasquale Mordasini, ${ }^{j}$ Johannes Kaesmacher, ${ }^{\mathrm{j}}$ Grégoire Boulouis, ${ }^{\mathrm{c}}$ on Behalf of the JENI Research \\ Collaborative \\ aDiagnostic and Therapeutic Neuroradiology, CHRU de Tours, Tours, France \\ ${ }^{b}$ Department of Interventional Neuroradiology, University Hospital Center of Montpellier, Gui de Chauliac Hospital, Montpellier, France \\ 'Centre Hospitalier Sainte Anne, Neuroradiology Department, Paris University, INSERM U1266, Psychiatry and Neurosciences Institute of Paris, \\ Paris, France \\ ¿University Hospital of Nancy, Department of Diagnostic and Therapeutic Neuroradiology, INSERM U1254, Nancy, France \\ 'Department of Diagnostic and Interventional Neuroradiology, Timone Hospital, Aix Marseille University, Marseille, France \\ ${ }^{f}$ Neurology Department, Timone Hospital, Aix Marseille University, Marseille, France \\ ${ }^{9}$ Department of Diagnostic and Interventional Neuroradiology, Guillaume et René Laennec University Hospital, Nantes, France

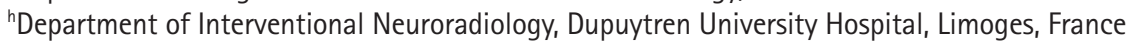 \\ 'Department of Diagnostic and Interventional Neuroradiology, Pellegrin Hospital-University Hospital of Bordeaux, Bordeaux, France \\ ${ }^{j}$ Institute of Diagnostic, Interventional and Pediatric Radiology and Institute of Diagnostic and Interventional Neuroradiology, University \\ Hospital Bern, Inselspital, University of Bern, Bern, Switzerland
}

Background and Purpose Patients with acute ischemic stroke, proximal vessel occlusion and a large ischemic core at presentation are commonly not considered for mechanical thrombectomy (MT). We tested the hypothesis that in patients with baseline large infarct cores, identification of remaining penumbral tissue using perfusion imaging would translate to better outcomes after MT.

Methods This was a multicenter, retrospective, core lab adjudicated, cohort study of adult patients with proximal vessel occlusion, a large ischemic core volume (diffusion weighted imaging volume $\geq 70 \mathrm{~mL}$ ), with pre-treatment magnetic resonance imaging perfusion, treated with MT (2015 to 2018) or medical care alone (controls; before 2015). Primary outcome measure was 3-month favorable outcome (defined as a modified Rankin Scale of 0-3). Core perfusion mismatch ratio (CPMR) was defined as the volume of critically hypo-perfused tissue (Tmax $>6$ seconds) divided by the core volume. Multivariable logistic regression models were used to determine factors that were independently associated with clinical outcomes. Outputs are displayed as adjusted odds ratio (aOR) and 95\% confidence interval (CI).

Results $A$ total of 172 patients were included (MT $n=130$; Control $n=42$; mean age $69.0 \pm 15.4$ years; $36 \%$ females). Mean core-volume and CPMR were $102.3 \pm 36.7$ and $1.8 \pm 0.7 \mathrm{~mL}$, respectively. As hypothesized, receiving MT was associated with increased probability of favorable outcome and functional independence, as CPMR increased, a difference becoming statistically significant above a mismatch-ratio of 1.72 . Similarly, receiving MT was also associated with favorable outcome in the subgroup of 74 patients with CPMR $>1.7$ (aOR, 8.12;
Correspondence: Grégoire Boulouis Centre Hospitalier Sainte Anne, Neuroradiology Department, Paris University, INSERM U1266, Psychiatry and Neurosciences Institute of Paris, 1 rue Cabanis, 75014 Paris, France

Tel: +33-145658574

Fax: +33-145658574

E-mail: gregoireboulouis@gmail.com

Received: October 25, 2019

Revised: March 30, 2020

Accepted: April 28, 2020 
95\% Cl, 1.24 to $53.11 ; P=0.028)$. Overall (prior to stratification by CPMR) $73(42.4 \%)$ patients had a favorable outcome at 3 months, with no difference amongst groups.

Conclusions In patients currently deemed ineligible for MT due to large infarct ischemic cores at baseline, CPMR identifies a subgroup strongly benefiting from MT. Prospective studies are warranted.

Keywords Acute stroke; Ischemic stroke; Thrombectomy; Endovascular treatment; Perfusion imaging

\section{Introduction}

In recent years, mechanical thrombectomy (MT) has demonstrated its compelling efficacy in reducing mortality and functional dependence for patients with acute ischemic stroke (AIS) due to anterior proximal vessel occlusion (PVO). ${ }^{1,2}$ Patients with an unfavorable imaging profile at baseline, assessed using magnetic resonance imaging (MRI) diffusion weighted imaging (DWI) volume core $(\geq 70 \mathrm{~mL}){ }^{1}$ or the computed tomography (CT)-based-Alberta Stroke Program Early CT score (ASPECTS) $<6$, were excluded in four of the seven randomized clinical trials that validated MT in AIS-PVO, ${ }^{3}$ precluding to draw strong conclusions in this subgroup. Hence they are typically not offered MT in clinical practice, ${ }^{1}$ despite converging evidence suggesting a benefit of MT despite large ischemic core (LIC), with almost $25 \%$ of patients experiencing favorable functional outcome after $\mathrm{MT}^{3-9}$

Perfusion imaging is used in the diagnostic work up of AIS to identify hypo-perfused yet not infarcted (e.g., 'at-risk' or 'salvageable') brain tissue, ${ }^{10}$ and to estimate the core perfusion mismatch ratio (CPMR). ${ }^{2}$ Nonetheless, perfusion data remain very scarce in patients with LIC before MT, while they are critically needed to pragmatically design future randomized trials, and better select patients for MT until then.

We hypothesized that perfusion imaging may enhance the effective selection of AIS-PVO patients with LIC by determining those likeliest to benefit from revascularization and tested this hypothesis in a cohort study using data from a multicenter cohort, by comparing the rates of favorable functional outcome, symptomatic intracerebral hemorrhage $(\mathrm{sICH})$, and case-fatality in patients with DWI assessed baseline LIC (>70 mL) and perfusion who received MT versus patients who were treated with intravenous thrombolysis only. The hypothesis driving this analysis was that in patients with baseline large infarct cores, identification of significant remaining penumbral tissue, as assessed using CPMR, would translate to better outcomes after MT.

\section{Methods}

\section{Study design}

The study was a multicenter, core-lab adjudicated, observational retrospective cohort study. The "Jeunes en Neuroradiologie Interventionnelle Research Collaborative" (JENI-RC), is a recently launched trainee-led research network. ${ }^{1 "}$ Local JENI-RC members were asked to provide de-identified data for patients with DWI ASPECTs 0-6 otherwise meeting study inclusion criteria (see below). Case report form items included demographics, relevant past medical history, $\mathrm{sICH}$, and 3-month functional outcome. Imaging data were centralized by the internal core lab, for central assessment. Nine academic centers contributed data (eight in France, and one in Switzerland). This report was prepared according to the Strengthening the Reporting of Observational Studies in Epidemiology (STROBE) statement. ${ }^{12}$

\section{Ethics}

As for all non-interventional retrospective studies of de-identified data in France, written informed consent was waived and a commitment to compliance (reference methodology CPMR3) was filed to the French National Information Science and Liberties Commission prior to data centralization, in respect to the General Data Protection Regulation.

\section{Inclusion criteria}

In the MT group, we included consecutive adult patients with AIS after 2015 if they had an occlusion of the intracranial internal carotid artery or of the M1 segment of the middle cerebral artery; had a large pretreatment ischemic core volume defined as $70 \mathrm{~mL}$ or more on magnetic resonance-DWI as assessed centrally; had no preexisting handicap as defined by a modified Rankin Scale (mRS) of 2 or higher; underwent MT; and if pre-treatment MR-T2* perfusion sequence had been performed. The control group was constituted by retrospectively querying the prospective intravenous tissue plasminogen activator (tPA) stroke data base at a single university hospital, to identify patients treated before MT related guidelines in 2015 
who met the same criteria but did not receive MT due to guidelines compliance.

\section{Imaging analysis and mismatch definition}

The post-processing and images interpretation were performed centrally after complete de-identification, by an internal corelab (B.K. and G.B.), blinded to clinical data.

Ischemic core volumes (DWI volume) were calculated using Olea-Sphere version 3.0 software (Olea Medical, La Ciotat, France), by the semi-automated segmentation of brain tissue ipsilateral to the occlusion with apparent diffusion coefficient of $0.6 \times 10^{-3} \mathrm{~mm}^{2} / \mathrm{sec}$ or less. Perfusion maps were generated using the Olea-Sphere version 3.0 software then used to calculate critically hypo-perfused brain tissue, with an inferior threshold set, according to recent literature, ${ }^{13}$ at $\mathrm{T} \max >6 \mathrm{sec}-$ onds. As in previous large studies investigating core penumbral mismatch, ${ }^{14,15}$ we defined penumbral tissue as the volume of critically hypo-perfused tissue minus the DWI volume. Similarly, we defined the CPMR, as the volume of critically hypo-perfused tissue divided by the DWI volume, and defined a target mismatch as patients with a CPMR of 1.8 or above.

\section{Assessment criteria}

The primary endpoint was functional outcome assessed at 3 months, using the mRS, with a favorable outcome defined by a score of 3 or less, taking into account the inherent severity of AIS with baseline LIC, and in line with recent literature. Secondary end points included functional independence defined as mRS of 0-2. Ninety days mortality, and the rate of symptomatic intracerebral hemorrhage within 7 days (according to European Cooperative Acute Stroke Study [ECASS] II criteria). ${ }^{16}$ Substantial reperfusion was defined as a modified Thrombolysis in Cerebral Infarction score of $2 \mathrm{~B}, 2 \mathrm{C}$, or $3 .^{17}$

\section{Statistical analysis}

Baseline characteristics of patients has been compared between MT group and control group. Continuous variables were summarized using mean \pm standard deviation or median (interquartile range) where appropriate, and discrete variables were summarized using counts (percentages). Chi-square test, Fisher-exact test, t-test, Mann-Whitney test were used as appropriate for the univariate analysis, with a $P<0.05$ as the threshold for statistical significance.

Clinical outcomes were compared between MT group and control group. First, multivariable logistic regression models were used to determine factors that were independently associated with clinical outcomes. Variables associated with the outcome in univariate analysis $(P \leq 0.1)$ were entered into nomi- nal logistic models, with a prespecified adjustment for age, infarct core, and CPMR. Backward elimination was then used to remove non-significant variables ( $P>0.05)$. The adjusted odds ratios $(\mathrm{aOR})$ and $95 \%$ confidence interval $(\mathrm{Cl})$ of having favorable outcome (model 1) and functional independence (model 2) were reported. Then, a sensitivity analysis was performed to assess for changes in the results in patients with a predefined CPMR cutoff of 1.8, as was reported in previous cornerstone studies analyzing the pertinence of CMPR in AIS-PVO patients. ${ }^{13,18}$ Finally, a shift analysis of mRS score (of 0-6 points) based on the proportional odds model has been performed. All analyses were done using JMP Pro 14 (Institute Inc., Cary, NC, USA) software. Where needed, we derived $95 \% \mathrm{Cl}$ by bootstrapping (5,000 occurrences) statistical results. ${ }^{18}$

\section{Results}

\section{Study population}

Between January 2015 and July 2018, in the MT group a total of 971 patients with AIS-PVO and ASPECTs 0-6 were screened for inclusion. After applying inclusion criteria, 168 patients were evaluated by the core lab, of which 130 were finally included and analyzed in the MT group. Before 2015, a total of 154 patients with DWI-ASPECTs 0-6 were screened for inclusion in the control group, and 42 met study criteria (Figure 1). A total of 172 were finally included in the present analysis (36\% females, mean age 69.0 \pm 15.4 years) (Table 1 for baseline clinical-imaging characteristics). Patients in the MT group were

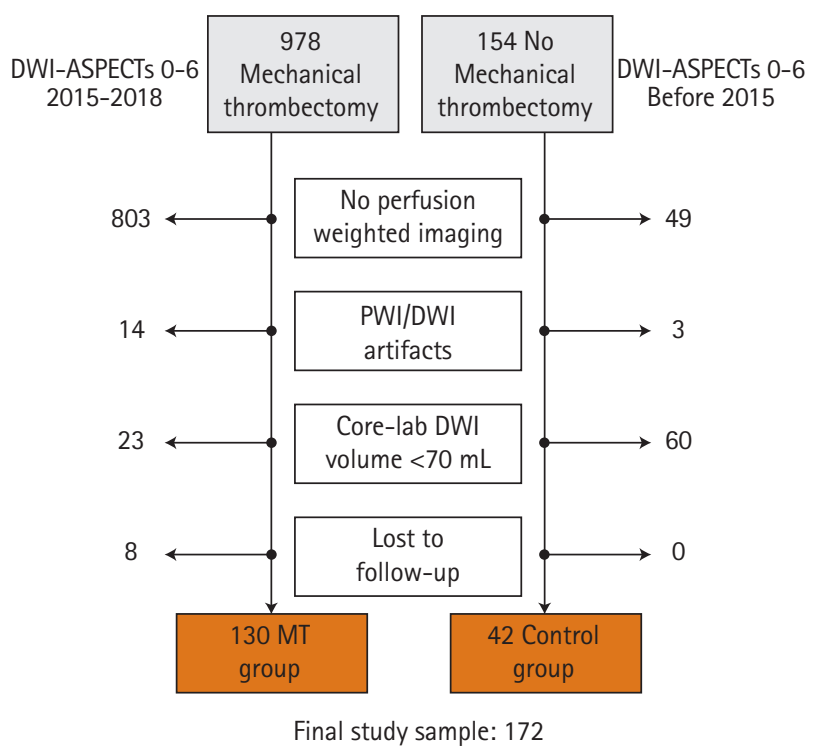

Figure 1. Flow chart. DWI, diffusion weighted imaging; ASPECTS, Alberta Stroke Program Early Computed Tomography Score; PWI, perfusion weighted imaging; MT, mechanical thrombectomy. 
more frequently females ( $41 \%$ vs. $21 \%, P=0.03$ ), less frequently received intravenous tPA ( $48.5 \%$ vs. $100 \%, P<0.01$ ), were more frequently referred from an outside primary stroke center hospital $(25.4 \%$ vs. $0 \%, P<0.01)$, were younger $(66.2 \pm 14.9$ yearsold vs. $77.7 \pm 13.5, P<0.01)$. They did not differ otherwise in terms of baseline clinical parameters, notably for delay between onset and qualifying imaging $(P=0.58)$ and occlusion site $(P=0.82)$. Substantial recanalization was achieved in $111 / 140$ patients in the MT group (79.3\%).

\section{Core and penumbra}

Mean core DWI volume was $102.3 \pm 36.7 \mathrm{~mL}$ and did not differ between groups. The volume of critically hypo-perfused tissue was larger in the MT group (mean $180.8 \pm 72.4 \mathrm{~mL}$ vs. $145.5 \pm 52.5$ $\mathrm{mL}, P<0.01$ ), which consequently demonstrated larger penumbral volumes (mean $76.8 \pm 63.7 \mathrm{~mL}$ vs. $48.8 \mathrm{ml} \pm 40.9 \mathrm{~mL}, P<0.01$ ) as well as higher CPMRS (mean $1.8 \pm 0.7$ vs. $1.5 \pm 0.5, P<0.01$ ). A total of 90 (52\%), 65 (38\%), and 53 (31\%) patients had CPMRS above $1.6,1.8$, and 2.0 respectively (Supplementary Figure 1, in the supplemental material for detailed CPMR distribution in the cohort).

\section{Outcomes}

\section{Functional outcomes}

At 3 months, 73 (42.4\%) patients had a favorable functional outcome (mRS 0-3), with no difference amongst groups prior to stratification by CPMR ( $43.1 \%$ in the MT vs. $40.5, P=0.86$ ). Forty-one patients $(23.8 \%)$ were functionally independent and 54 (31.4\%) were deceased ( $P=1.000)$ (Table 2). Unfavorable outcome was associated with higher age, higher baseline NIHSS, history of hypertension, and diabetes mellitus (Supplementary Table 1).

Independent of, as well as within, treatment groups both larger core and larger penumbral volumes were associated with

Table 1. Baseline characteristics of included patients

\begin{tabular}{|c|c|c|c|c|}
\hline Variable & All $(n=172)$ & Control $(n=42)$ & MT $(n=130)$ & $P$ \\
\hline Age $(y r)$ & $69.0 \pm 15.4$ & $77.7 \pm 13.5$ & $66.2 \pm 15$ & $<0.001$ \\
\hline Female sex & $62(36)$ & $9(21.4)$ & $53(40.8)$ & 0.029 \\
\hline Dyslipidemia & $69(40.1)$ & $18(42.9)$ & $51(39.2)$ & 0.718 \\
\hline Diabetes mellitus & $26(15.1)$ & $7(16.7)$ & $19(14.6)$ & 0.811 \\
\hline Tobacco use (current or past) & $56(32.6)$ & 14 (33.3) & $42(32.3)$ & 1.000 \\
\hline Hypertension & $97(56.4)$ & $24(57.1)$ & 73 (56.2) & 1.000 \\
\hline NIHSS & $18.5 \pm 4.5$ & $18 \pm 5.1$ & $18.7 \pm 4.2$ & 0.435 \\
\hline Left sided stroke & $75(43.6)$ & $16(38.1)$ & $59(45.4)$ & 0.476 \\
\hline iv $\mathrm{tPA}$ & $105(61)$ & $42(100)$ & $63(48.5)$ & $<0.001$ \\
\hline Drip and ship & $33(19.2)$ & $0(0)$ & $33(25.4)$ & $<0.001$ \\
\hline ICA occlusion & $31(18)$ & $7(16.7)$ & $24(18.5)$ & 0.269 \\
\hline Delay till imaging (min) & $161.3 \pm 129$ & $152.7 \pm 108.5$ & $164 \pm 135.2$ & 0.583 \\
\hline Volume T $\max <6 \mathrm{sec}(\mathrm{mL})$ & $172.2 \pm 69.6$ & $145.5 \pm 52.5$ & $180.8 \pm 72.4$ & $<0.001$ \\
\hline Mismatch ratio & $1.8 \pm 0.7$ & $1.5 \pm 0.5$ & $1.8 \pm 0.7$ & $<0.001$ \\
\hline Core volume $(\mathrm{mL})$ & $102.3 \pm 36.7$ & $96.7 \pm 33$ & $104 \pm 37.7$ & 0.233 \\
\hline
\end{tabular}

Values are presented as mean \pm standard deviation or number (\%).

MT, mechanical thrombectomy; NIHSS, National Institute of Health Stroke Scale; iv tPA, intravenous tissue plasminogen activator; ICA, internal carotid artery.

Table 2. Outcome of included patients

\begin{tabular}{|c|c|c|c|c|}
\hline Variable & All $(n=172)$ & Control $(n=42)$ & MT $(n=130)$ & $P$ \\
\hline Substantial recanalization & - & - & $111(79.3)$ & \\
\hline 90-day, mRS 0-2 & 41 (23.8) & $11(26.2)$ & $30(23.1)$ & 0.684 \\
\hline 90-day, mRS 0-3 & $73(42.4)$ & $17(40.5)$ & $56(43.1)$ & 0.857 \\
\hline 90-day, mortality & 54/172 (31.4) & 13 (30.9) & $41(31.5)$ & 1 \\
\hline $\mathrm{sICH}$ & 31/161 (19.3) & $5 / 35(14.3)$ & $26 / 126(20.26)$ & 0.483 \\
\hline
\end{tabular}

Values are presented as number (\%).

MT, mechanical thrombectomy; mRS, modified Rankin Scale; sICH, symptomatic intra-cranial hemorrhage. 
poor functional outcome in univariable analysis $(185 \pm 67.9 \mathrm{~mL}$ in patients with $\mathrm{mRS} 4-6$ vs. $154 \pm 68.4 \mathrm{~mL}$ in those with $\mathrm{mRS}$ $0-3, P<0.01$; and $76 \pm 59$ vs. $67 \pm 60, P=0.04$ ) (Figure 2 ).

In the entire population, there was no difference in favorable outcome between treatment groups ( $P=0.68)$ (Table 1). After adjusting for age, hypertension, diabetes, core volume, CPMR, delay to imaging, intravenous IPA before MT, and occlusion site, there was a significant interaction between MT effect and CPMR in both models (Model 1: aOR, 0.29; $P=0.008$; and Model $2: \mathrm{aOR}, 0.4 ; P=0.047)$, indicating an increasing benefit of MT as CPMR increases (Table 3). In the same model, with mismatch ratio dichotomized as a nominal variable (below or above 1.8) the odds ratio of the interaction term between treatment arm
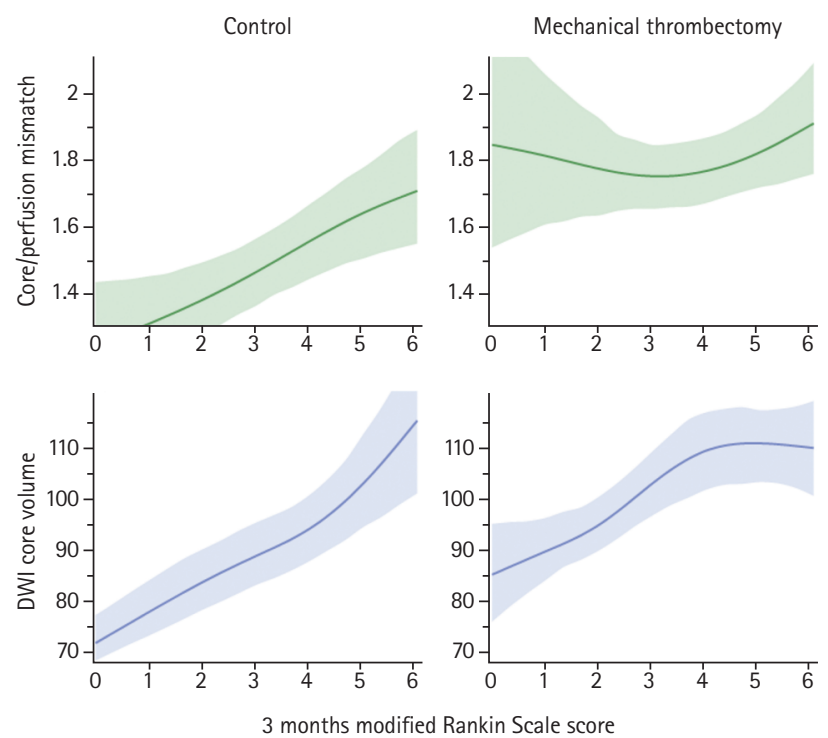

Figure 2. Modified Rankin Scale at 3 months and initial diffusion weighted imaging (DWI) core volume and mismatch ratio per group. and mismatch ratio (>1.8), was $0.42(95 \% \mathrm{Cl}, 0$ to 0,82 ; $P=0.02$ ).

Analyzing the probability for favorable outcome in the fully adjusted model plotted against CPMR, we showed that receiving MT (vs. not receiving MT) was associated with increased probability of favorable outcome and functional independence, as CPMR increased, a difference becoming statistically significant above a CPMR of 1.72 for favorable outcome, and above 1.93 for functional independence (Figure 3).

As a sensitivity analysis, when stratifying by CPMR; in the sample of 65 patients with a CPMR of 1.8 or above, after adjusting for group specific outcome predictors (age, core volume, intravenous $\mathrm{PAA}$, and CPMR), receiving MT was associated with a significant increase in the rate of favorable outcome (aOR, 999; 95\% Cl, 999 to infinite). Similarly, in the subgroup of 74 patients for which CPMR exceeded 1.7 (42\% of favorable outcome in the MT group vs. $20 \%$ in the control group, $P=0.031$; receiving MT was associated with a significantly increased rate of favorable outcome) (aOR, 8.12; 95\% Cl, 1.24 to 53.11, $P=0.028$ ). Using ordinal regression, receiving MT was associated with overall favorable shift in mRS distribution (common risk ratio, $1.83 ; 95 \% \mathrm{Cl}, 1.01$ to $3.44 ; P=0.049$ ) (Figure 4).

Finally, when restricting the sample to patients in the MT group, we found substantial recanalization to be associated with significantly higher odds of favorable outcome and of 3 months functional independence, in fully adjusted models (aOR, 53.7; $95 \% \mathrm{Cl}, 5.0$ to 573; $P<0.001$; and aOR infinite, $P<0.001$, respectively). Lower age $(P<0.001)$, lower mismatch ratio $(P=0.03)$, and lower core volume $(P<0.001)$ were also associated with higher odds of favorable outcome, but the interaction between CPMR and substantial recanalization only tended towards significance $(P=0.058)$.

Table 3. Multivariable models for outcome

\begin{tabular}{|c|c|c|c|c|c|c|}
\hline \multirow{2}{*}{ Variable } & \multicolumn{3}{|c|}{ For favorable outcome } & \multicolumn{3}{|c|}{ For functional independence } \\
\hline & $\mathrm{aOR}$ & $95 \% \mathrm{Cl}$ & $P$ & $\mathrm{aOR}$ & $95 \% \mathrm{Cl}$ & $P$ \\
\hline Age (yr) & 0.95 & $0.92-0.98$ & $<0.001$ & 0.97 & $0.94-1.00$ & 0.048 \\
\hline Diabetes & 0.33 & $0.11-1.02$ & 0.049 & 0.36 & $0.09-1.45$ & 0.015 \\
\hline Hypertension & 0.85 & $0.39-1.82$ & 0.673 & 0.79 & $0.33-1.88$ & 0.591 \\
\hline iv $\mathrm{tPA}$ & 1.74 & $0.76-3.96$ & 0.188 & 2.10 & $0.83-5.36$ & 0.119 \\
\hline Delay till imaging (min) & 1.00 & $1.00-1.00$ & 0.934 & 1.00 & $1.00-1.00$ & 0.754 \\
\hline ICA occlusion & 0.75 & $0.28-2.00$ & 0.572 & 0.59 & $0.18-1.94$ & 0.391 \\
\hline Volume core (for each $10 \mathrm{~mL}$ ) & 0.79 & $0.69-0.89$ & $<0.001$ & 0.74 & $0.62-0.89$ & $<0.001$ \\
\hline Core perfusion mismatch (per unit) & 0.19 & $0.06-0.56$ & $<0.001$ & 0.34 & $0.12-0.97$ & 0.026 \\
\hline Received MT & 1.95 & $0.62-6.07$ & 0.248 & 1.64 & $0.48-5.58$ & 0.426 \\
\hline Interaction mismatch group & - & - & 0.009 & - & - & 0.047 \\
\hline
\end{tabular}

aOR, adjusted odds ratio; $\mathrm{Cl}$, confidence interval; iv $\mathrm{PA}$, intravenous tissue plasminogen activator; ICA, internal carotid artery; MT, mechanical thrombectomy. 

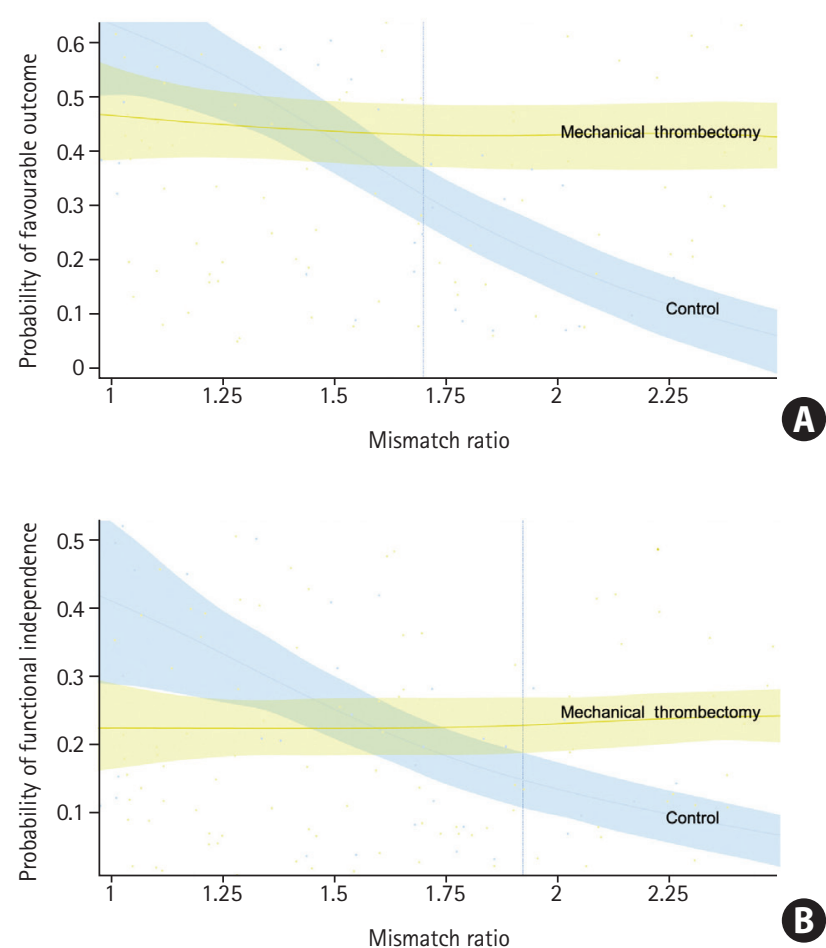

Figure 3. Probability of favourable functional outcome (A) or functional independence (B) by mismatch ratio, in patient receiving mechanical thrombectomy (yellow), and in the control group (blue).
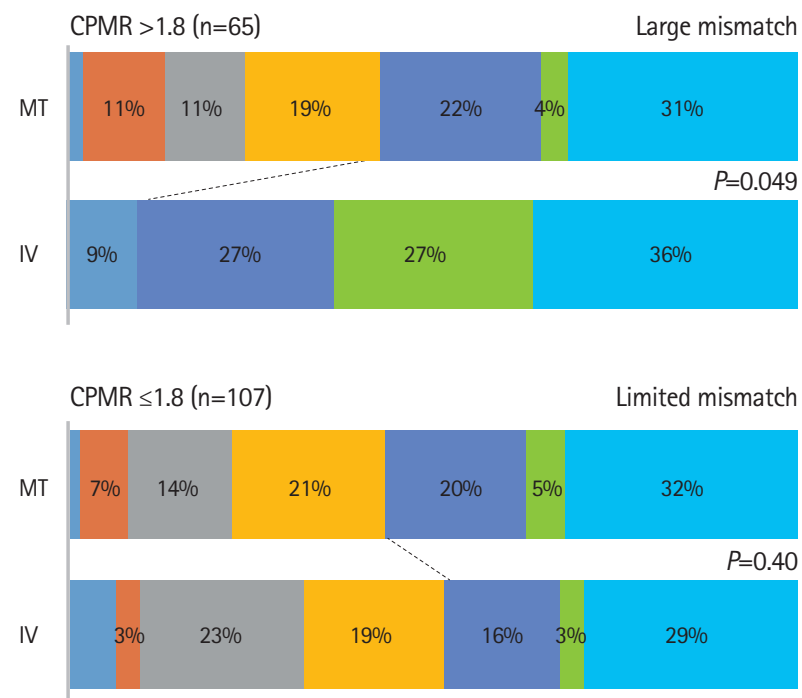

mRS scores $\square 0 \square 1 \square 2 \square 3 \square 4 \square 5 \square 6$

Figure 4. Modified Rankin Scale (mRS) distribution in patients receiving mechanical thrombectomy (MT) and those not receiving MT, stratified by core perfusion mismatch ratio (CPMR). IV, intravenous.

\section{Secondary outcomes}

At 3 months, 54 patients were deceased (31.5\% in the MT vs. $30.9 \%$ in the control group, $P=1$ ). Fully adjusted model identi- fied older age, larger core volume, higher CPMR, and diabetes mellitus as being associated with significantly higher mortality (all $P<0.01$ ) (Table 2). Treatment group did not influence 3 months mortality rate (Supplementary Tables 2 and 3 ).

A total of 31 of 161 patients with available data suffered from a sICH (20.6\% in the MT group vs. $14.3 \%, P=0.48$ ) (Table 2). In our sample, receiving MT was not associated with higher odds of sICH (aOR, 1.94; $95 \% \mathrm{Cl}, 0.6$ to $6.26 ; P=0.26)$. While larger infarct core was associated with more frequent $\mathrm{sICH}$ (aOR, 1.01 for each $\mathrm{mL}$ increase in core volume; $95 \% \mathrm{Cl}, 1$ to 1.03; $P=0.02$ ), higher CPMR was not (Supplementary Tables 4 and 5). In the MT group, substantial recanalization was not associated with decreased odds of $\mathrm{sICH}(P=0.49)$.

\section{Discussion}

In this multicenter collaborative study we showed that (1) MT is strongly beneficial on clinical outcome of patients with large infarct core at baseline, and persisting core/penumbral mismatch (CPMR $>1.72$ in our sample and by extension $>1.8$ ), with no heterogeneity in treatment effect across strata of CPMR and (2) that MT does not increase the odds of $\mathrm{sICH}$ and mortality.

The question of the best treatment approach for AIS-PVO patients with large infarct at baseline remains unanswered, despite being amongst the most timely and relevant in acute stroke care. There is indeed a critical need to assess treatment opportunities expansion to those patients that were excluded from princeps MT trials (especially patients with LIC, that are at critical risk of poor functional outcomes and for which guidelines remain ambiguous), and may in turn not be offered MT, despite a potential benefit. Until the results of dedicated trials such as Exploration of the limits of mechanical thrombectomy indications in a single action-Large Stroke Therapy Evaluation (IN EXTREMIS-LASTE) or Efficacy and Safety of Thrombectomy in Stroke With Extended Lesion and Extended Time Window (TENSION) become available, the community faces a challenge in the treatment strategy for this subgroup.

Expectedly, our results are in line with previous studies ${ }^{3,4,7,19-21}$ demonstrating the direct correlation between larger infarct core sizes decreased odds of favorable functional outcome. In our sample as a whole, for every $10 \mathrm{~mL}$ increase in core volume, there was a $22 \%$ increase in the risk of unfavorable outcome, and a $26 \%$ increase in the risk of 90 days functional dependence. Our results, in that sense, confirm that presenting with a large infarct at baseline is of poorer prognosis and do support careful expectations' management with families and caregivers.

Less intuitively, but substantiating our working hypothesis, increasing CPMR was also associated with lower chance of favor- 
able outcome, with an odd decrease of 81\% for every mismatch unit increase. This finding was not unexpected, since patients with higher CPMRS are at inherent higher risk for infarct progression within the hypo-perfused area, until recanalization or if recanalization doesn't occur, or not fast enough. In our sample, the benefit of MT over the control group became significant above a CPMR of 1.72 corresponding to a minimal penumbral volume of $50.4 \mathrm{~mL}$ (e.g., if the patient has a core volume of exactly $70 \mathrm{~mL}$ ) and was stable in the stratified subgroup of patients with CPMRS above 1.7. Although the conceptual framework supporting the benefit of MT in patients with PVO and target mismatch has been substantiated by a large number of publications, including the cornerstone studies from the diffusion and perfusion imaging evaluation for understanding stroke evolution (DEFUSE) group, ${ }_{1}^{13,22}$ there is still limited dedicated studies in the specific subgroup of patients with larger infarcts at baseline. Rebello et al. ${ }^{5}$ demonstrated in a sample of 24 patients with large infarct cores assessed using CT perfusion (cerebral blood flow $<30 \% ; 70 \mathrm{~mL}$ ) and an penumbra volumes (Tmax $>6$ seconds) above 40 to $50 \mathrm{~mL}$ that MT was associated with significant reduction in final infarct volumes $(110 \pm 65 \mathrm{~mL}$ vs. $319 \pm 147 \mathrm{~mL}, P<0.001$ ) but only a nonsignificant improvement in the overall distribution of $m R S$ scores favoring the treatment group $(P=0.18)$. These neutral results with regards to clinical outcome, are likely due to insufficient power, in this subgroup limited by a binary design that excluded patients with limited mismatch, precluding to further test interaction between MT effects and CPMR. More recently, Campbell et al. ${ }^{15}$ showed in post hoc analyses of individual patient level data from The Highly Effective Reperfusion evaluated in Multiple Endovascular Stroke Trials (HERMES) collaboration, that amongst the 583 patients with computed tomography perfusion (CTP), the interaction between CPMR and endovascular treatment effect was not significant $(P=0.15)$, but statistical power was strongly limited by the small number of patients not meeting criteria (less than 5\% with a CPMR $<1.8$ amongst the 583 patients with CTP). In this study, CTP mismatch volume was negatively associated, in univariate analysis, with functional improvement (common odds ratio per $10 \mathrm{~mL} 0.96 ; 95 \%$ $\mathrm{Cl}, 0.93$ to $0.99 ; P=0.009$ ) reinforcing the conceptual balance paradox by which larger mismatch volumes are associated with decreased favorable outcomes due to increased possibilities of infarct progression, and in turn explaining the increasing benefit of MT as CPMR increases, a notion that had not been confirmed before our study in patients with larger infarct cores.

In secondary analyses, we did not show a significant association between MT and the risk of sICH, and there was conversely a positive interaction between infarct core volume and treatment group, in favor of MT. There's been several reports on the risk of $\mathrm{SICH}$ after MT in $\mathrm{LIC}$, none of which showed an increase in the risk of sICH after MT except in the HERMES collaboration ${ }^{3}$ where, for patients with ASPECTS 0-4, sICH was more frequent in the MT group, although not significantly (adjusted COR, 3.94; $95 \% \mathrm{Cl}, 0.94$ to 16.49 ; $P$ interaction $=0.025$ ), and not reproduced when restricting the sample to patients with DWI volume $\geq 70$ $\mathrm{mL}$ were no significant difference of sICH between EVT (1/23, $4.3 \%)$ and best medical treatment $(2 / 37,5.4 \%)$ was found. ${ }^{3,15}$ Of critical note, the most important predictor of $\mathrm{sICH}$ and parenchymal hemorrhage is core volume, independent of treatment modality, 3,7,19,20,23 likely explaining that successful reperfusion was associated with lower sICH in many "real life" recent studies. ${ }^{7,19,20}$ Whether the benefits of not revascularizing a patient to prevent $\mathrm{sICH}$, outweigh those of revascularization to prevent infarction extension is unknown, but very unlikely, especially in patients with important mismatch, at highest risk for infarct progression and progression to malignant infarction. Our study was neither powered nor designed to answer this question.

There's a long ongoing debate on optimal imaging modality (CT or MRI) for AIS-PVO patients' selection for revascularization strategies. ${ }^{24-26}$ The main risk of patients' selection in the context of AIS is over-selection, that is, to decline a patient a treatment that may have been beneficial. For patients with LIC, the question of over-selection is amongst the timeliest in modern stroke care. The first level of over-selection may happen at the core assessment level (e.g., dismissing a patient because of large infarct). Interestingly, in the HERMES collaboration, ${ }^{3}$ the treatment benefit in patients with ASPECTs 0-4 derived from the aggregation of CT and MRI ASPECTs data, and was likely contingent on the disproportionately larger effect size seen in the MRI subgroup (aOR, 3.57; 95\% Cl, 1.22 to 10.39 vs. aOR, $1.68 ; 95 \% \mathrm{Cl}$, 0.58 to 4.87 in the CT group). Similarly, Campbell et al. ${ }^{15}$ demonstrated that CTP was associated with significantly halved proportion of patients functional independence $(\mathrm{OR}, 0.47 ; 95 \% \mathrm{Cl}, 0.30$ to $0.72 ; P=0.0007$ ), and also with less functional improvement ( $\mathrm{COR}, 0.51 ; 95 \% \mathrm{Cl}, 0.36$ to $0.72 ; P=0.0001$ ) when compared to $\mathrm{MRI}$, both studies reinforcing the notion that MRI may better select patients for MT, but at the inherent risk of overselecting. In that sense, our study provides critical answers with regards to outcome in patients with LIC by the use of MRI with known higher conspicuity and reproducibility to detect and measure infarct core when compared to CT (especially when ASPECTs is used). ${ }^{27}$ The second level of over-selection, accounting that patients are considered for MT even with a LIC, is perfusion imaging. Our study showed, using strict post processing method, that patients with a CPMR above 1.72 (and by extension, over 1.8) demonstrated more favorable outcomes when treated with MT but the benefit increase was expectedly linear and not sudden at a discrete threshold of 1.72 . While this finding suggests that pa- 
tients with lower CPMR are likely to also benefit from MT, more subjects would be needed to demonstrate it, the benefit would likely be lesser, and this would need to be confirmed in a larger scale study. Importantly, we did not demonstrate any harm derived from MT in patients with lower CPMR.

Altogether, by comforting patho-physiologically plausible and statistically stable answers the question of the benefit of MT in patients with LIC at baseline and persisting salvageable tissue, our study raises ethical and philosophical considerations. There is growing evidence that by withholding MT by fear of potential harm for some patients, we may actually dismiss a much larger of patients that may have benefited from revascularization. In that sense, by demonstrating the continuum of unfavorable outcome paralleling the increases in core as well as mismatch volumes, and by showing the increasing benefit of MT with increasing CPMR, we actually fuel the argument that perfusion imaging is not needed as it may delay revascularization in eligible patients (and be used to decline MT to patients with CPMRS in the lower ranges, despite potential benefit). With no evidence of harm from MT in any of the explored configurations in our sample and the above-mentioned continuums in treatment benefit increases, it may be reasonable not to withhold treatment based on strict-cut offs (e.g., the study specific 1.72, applicable only to our sample, or the more common 1.8), but to adjust treatment decisions to both outcome and patients/families' centered expectations. Results from future trials may yield more definite answers to these questions, although the authors are not aware of any ongoing large randomized study using perfusion imaging as a selection criterion in patients with LIC.

Our study has limitations, most inherent to its design. It was a retrospective analysis, with a high risk of selection bias in included cases and important number of excluded cases due to the limited penetration of perfusion imaging for AIS amongst French centers and to the yet unusual use of MT in patients with LIC. For similar reasons, our sample size did not allow for a split into a derivation and a validation cohort, but we aimed at substantiating our estimates by various sensitivity analyses, which proved to be stable. We acknowledge that our control group was biased, by the fact that it included only patients who received intravenous $\mathrm{PAA}$, and that this bias may have yielded underestimated estimates of the benefit of MT over best medical management. Lastly, using CPMR instead of CT allowed for more precise estimates of ischemic infarct cores, but make our results less generalizable beyond the pathophysiological rationale it provides.

\section{Conclusions}

In patients currently deemed ineligible for MT due to large infarct ischemic cores at baseline, CPMR identifies patients strongly benefiting from MT. These finding provide a data-driven framework supporting both the relevance of CPMR perfusion assessment at the acute phase of AIS due to PVO in patients with LIC, and the notion that there is no strict plausible cutoff in core or penumbral volumes above or below which MT may become harmful by comparison to best medical treatment alone. Our results may help at informing the design of future randomized trials and may, further, help inform clinical practice for more individualized decision making in this subgroup until higher level evidence becomes available.

\section{Supplementary materials}

Supplementary materials related to this article can be found online at https://doi.org/10.5853/jos.2019.02908.

\section{Disclosure}

The authors have no financial conflicts of interest.

\section{References}

1. Powers WJ, Rabinstein AA, Ackerson T, Adeoye OM, Bambakidis NC, Becker K, et al. 2018 Guidelines for the early management of patients with acute ischemic stroke: a guideline for healthcare professionals from the American Heart Association/ American Stroke Association. Stroke 2018;49:e46-e110.

2. Turc $G$, Bhogal $P$, Fischer $U$, Khatri $P$, Lobotesis $K$, Mazighi $M$, et al. European Stroke Organisation (ESO)-European Society for Minimally Invasive Neurological Therapy (ESMINT) guidelines on mechanical thrombectomy in acute ischemic stroke. J Neurointerv Surg 2019;11:535-538.

3. Román $L S$, Menon BK, Blasco J, Hernández-Pérez $M$, Dávalos $A$, Majoie CBLM, et al. Imaging features and safety and efficacy of endovascular stroke treatment: a meta-analysis of individual patient-level data. Lancet Neurol 2018;17:895-904.

4. Desilles JP, Consoli A, Redjem H, Coskun O, Ciccio G, Smajda $\mathrm{S}$, et al. Successful reperfusion with mechanical thrombectomy is associated with reduced disability and mortality in patients with pretreatment diffusion-weighted imaging-alberta stroke program early computed tomography score $\leq 6$. Stroke 2017:48:963-969.

5. Rebello LC, Bouslama M, Haussen DC, Dehkharghani S, Grossberg JA, Belagaje $\mathrm{S}$, et al. Endovascular treatment for patients 
with acute stroke who have a large ischemic core and large mismatch imaging profile. JAMA Neuro/ 2017;74:34-40.

6. Gautheron V, Xie Y, Tisserand M, Raoult H, Soize S, Naggara 0 , et al. outcome after reperfusion therapies in patients with large baseline diffusion-weighted imaging stroke lesions: a THRACE trial (mechanical thrombectomy after intravenous alteplase versus alteplase alone after stroke) subgroup analysis. Stroke 2018;49:750-753.

7. Gilgen MD, Klimek D, Liesirova KT, Meisterernst J, KlingerGratz PP, Schroth G, et al. Younger stroke patients with large pretreatment diffusion-weighted imaging lesions may benefit from endovascular treatment. Stroke 2015;46:2510-2516.

8. Mourand I, Abergel E, Mantilla D, Ayrignac X, Sacagiu T, Eker $O F$, et al. Favorable revascularization therapy in patients with ASPECTS $\leq 5$ on DWI in anterior circulation stroke. J Neurointerv Surg 2018;10:5-9.

9. Sarraj A, Hassan AE, Savitz S, Sitton C, Grotta J, Chen P, et al. Outcomes of endovascular thrombectomy vs medical management alone in patients with large ischemic cores: a secondary analysis of the optimizing patient's selection for endovascular treatment in acute ischemic stroke (SELECT) study. JAMA Neurol 2019;76:1147-1156.

10. Shih $L C$, Saver $J$, Alger JR, Starkman $S$, Leary MC, Vinuela $F$, et al. Perfusion-weighted magnetic resonance imaging thresholds identifying core, irreversibly infarcted tissue. Stroke 2003;34:1425-1430.

11. JENI Research Collaboration. A call for junior interventional neuroradiologists to join the JENI-Research Collaboration. J Neuroradiol 2018;45:341-342.

12. von Elm E, Altman DG, Egger M, Pocock SJ, Gøtzsche PC, Vandenbroucke JP, et al. The Strengthening the reporting of observational studies in epidemiology (STROBE) statement: guidelines for reporting observational studies. Lancet 2007;370:14531457.

13. Lansberg MG, Straka M, Kemp S, Mlynash M, Wechsler LR, Jovin TG, et al. MRI profile and response to endovascular reperfusion after stroke (DEFUSE 2): a prospective cohort study. Lancet Neurol 2012;11:860-867.

14. Goyal M, Menon BK, van Zwam WH, Dippel DW, Mitchell PJ, Demchuk AM, et al. Endovascular thrombectomy after largevessel ischaemic stroke: a meta-analysis of individual patient data from five randomised trials. Lancet 2016;387:1723-1731.

15. Campbell BCV, Majoie CBLM, Albers GW, Menon BK, Yassi N, Sharma $G$, et al. Penumbral imaging and functional outcome in patients with anterior circulation ischaemic stroke treated with endovascular thrombectomy versus medical therapy: a meta-analysis of individual patient-level data. Lancet Neurol
2019;18:46-55.

16. Fiorelli M, Bastianello S, von Kummer R, del Zoppo GJ, Larrue V, Lesaffre $E_{1}$ et al. Hemorrhagic transformation within 36 hours of a cerebral infarct: relationships with early clinical deterioration and 3-month outcome in the European Cooperative Acute Stroke Study I (ECASS I) cohort. Stroke 1999;30:2280-2284.

17. Goyal M, Fargen KM, Turk AS, Mocco J, Liebeskind DS, Frei D, et al. $2 \mathrm{C}$ or not $2 \mathrm{C}$ : defining an improved revascularization grading scale and the need for standardization of angiography outcomes in stroke trials. J Neurointerv Surg 2014;6:83-86.

18. Efron B, Tibshirani RJ. An Introduction to the Bootstrap. Boca Raton, FL: CRC Press, 1994.

19. Kaesmacher J, Chaloulos-lakovidis P, Panos L, Mordasini P, Michel P, Hajdu SD, et al. Mechanical thrombectomy in ischemic stroke patients with alberta stroke program early computed tomography score 0-5. Stroke 2019;50:880-888.

20. Panni P, Gory B, Xie Y, Consoli A, Desilles JP, Mazighi M, et al. Acute stroke with large ischemic core treated by thrombectomy. Stroke 2019;50:1164-1171.

21. Phan $K$, Saleh $S$, Dmytriw AA, Maingard J, Barras $C$, Hirsch $J A$, et al. Influence of ASPECTS and endovascular thrombectomy in acute ischemic stroke: a meta-analysis. J Neurointerv Surg 2019;11:664-669.

22. Albers GW, Marks MP, Kemp S, Christensen S, Tsai JP, OrtegaGutierrez $\mathrm{S}$, et al. Thrombectomy for stroke at 6 to 16 hours with selection by perfusion imaging. N Engl J Med 2018;378: 708-718.

23. Marsh $E B$, Llinas $R H$, Schneider $A L$, Hillis $A E$, Lawrence $E$, Dziedzic $P$, et al. Predicting hemorrhagic transformation of acute ischemic stroke: prospective validation of the HeRS score. Medicine (Baltimore) 2016;95:e2430.

24. Albers GW. Endovascular thrombectomy in patients with large infarctions: reasons for restraint. Lancet Neurol 2018;17:836837.

25. Kim JT, Cho BH, Choi KH, Park MS, Kim BJ, Park JM, et al. Magnetic resonance imaging versus computed tomography angiography based selection for endovascular therapy in patients with acute ischemic stroke. Stroke 2019;50:365-372.

26. Provost C, Soudant M, Legrand L, Ben Hassen W, Xie Y, Soize S, et al. Magnetic resonance imaging or computed tomography before treatment in acute ischemic stroke. Stroke 2019;50:659664.

27. Farzin $B$, Fahed R, Guilbert F, Poppe AY, Daneault N, Durocher $A P$, et al. Early $C T$ changes in patients admitted for thrombectomy: intrarater and interrater agreement. Neurology 2016;87:249-256. 
Supplementary Table 1. Univariate analysis of outcome predictors (mRS 0-3)

\begin{tabular}{lccc}
\hline Variable & Unfavorable $(n=99)$ & Favorable $(\mathrm{n}=73)$ & $P$ \\
\hline Age (yr) & $72.9 \pm 14.2$ & $63.8 \pm 15.5$ & $<.001$ \\
Female sex & $36(36.4)$ & $26(35.6)$ & 1 \\
Dyslipidemia & $49(49.5)$ & $20(27.4)$ & 0.003 \\
Diabetes mellitus & $20(20.2)$ & $6(8.2)$ & 0.005 \\
Tobacco use (current or past) & $28(28.3)$ & $28(38.4)$ & 0.187 \\
Hypertension & $65(65.7)$ & $32(43.8)$ & 0.005 \\
NIHSS & $19.3 \pm 4.5$ & $17.5 \pm 4.3$ & 0.011 \\
Left sided stroke & $46(46.5)$ & $29(39.7)$ & 0.355 \\
iv tPA & $56(56.6)$ & $49(67.1)$ & 0.205 \\
Drip and ship & $22(22.2)$ & $11(15.1)$ & 0.327 \\
ICA occlusion & $21(21.2)$ & $11(15.1)$ & 0.329 \\
Delay till imaging $(\mathrm{min})$ & $167.3 \pm 137.9$ & $153.1 \pm 116.4$ & 0.468 \\
Volume T max $<6$ sec $(\mathrm{mL})$ & $185.1 \pm 68$ & $154.7 \pm 68.4$ & $1.7 \pm 0.6$ \\
Mismatch ratio & $1.8 \pm 0.7$ & $94 \pm 26.6$ & 0.004 \\
Core volume $(\mathrm{mL})$ & $108.4 \pm 41.7$ & $56(76.7)$ & 0.166 \\
Received MT & $74(74.7)$ & & $<0.001$ \\
\hline Values are presented & & 0.851 \\
\end{tabular}

Values are presented as mean \pm standard deviation or number $(\%)$.

mRS, modified Rankin Scale; NIHSS, National Institute of Health Stroke Scale; iv tPA, intravenous tissue plasminogen activator; ICA, internal carotid artery; MT, mechanical thrombectomy.

Supplementary Table 2. Univariable analysis of 90-day mortality

\begin{tabular}{|c|c|c|c|}
\hline Variable & Dead $(n=54)$ & Alive $(n=118)$ & $P$ \\
\hline Age (yr) & $77.63 \pm 11.99$ & $65.09 \pm 15.21$ & $<0.001$ \\
\hline Female sex & 19 (35.2) & 43 (36.4) & 1 \\
\hline Dyslipidemia & $27(50)$ & $42(35.6)$ & 0.094 \\
\hline Diabetes & 15 (27.8) & $11(9.3)$ & 0.003 \\
\hline Hypertension & $37(68.5)$ & $60(50.8)$ & 0.033 \\
\hline NIHSS & $19.78 \pm 4.7$ & $17.96 \pm 4.25$ & 0.019 \\
\hline Tobacco use & $11(20.4)$ & $45(38.1)$ & 0.023 \\
\hline ICA occlusion & $10(18.5)$ & 22 (18.6) & 1.000 \\
\hline Delay till imaging (min) & $164.87 \pm 155.96$ & $159.61 \pm 115.33$ & 0.825 \\
\hline Drip and ship & $12(22.2)$ & $21(17.8)$ & 0.534 \\
\hline iv tPA & $30(55.6)$ & $75(63.6)$ & 0.401 \\
\hline Volume core (mL) & $111.3 \pm 43.4$ & $98.11 \pm 32.49$ & 0.025 \\
\hline Volume T max >6 sec (min) & $192.02 \pm 64.11$ & $163.13 \pm 70.38$ & 0.009 \\
\hline Mismatch ratio & $1.86 \pm 0.75$ & $1.7 \pm 0.65$ & 0.173 \\
\hline MT & $41(31.5)$ & $89(68.5)$ & vs. control, 1.000 \\
\hline Control & 13 (30.9) & $29(69.1)$ & \\
\hline
\end{tabular}

Values are presented as number (\%) or mean \pm standard deviation.

NIHSS, National Institute of Health Stroke Scale; ICA, internal carotid artery; iv tPA, intravenous tissue plasminogen activator; MT, mechanical thrombectomy. 
Supplementary Table 3. Multivariable model of 90-day mortality predictors (pre specified adjustment for core volume, mismatch ratio, and treatment group)

\begin{tabular}{lllc}
\hline Variable & aOR & $95 \% \mathrm{Cl}$ & \multicolumn{1}{c}{ P } \\
\hline Age & 1.11 & $1.07-1.16$ & 0.001 \\
Diabetes & 4.34 & $1.54-12.27$ & 0.006 \\
Volume core (each 10 mL) & 1.32 & $1.16-1.53$ & $<0.001$ \\
Core perfusion mismatch & 2.38 & $1.26-4.47$ & 0.006 \\
MT vs. Control & 0.49 & $0.19-1.37$ & 0.177
\end{tabular}

$\mathrm{aOR}$, adjusted odds ratio; $\mathrm{Cl}$, confidence interval; $\mathrm{MT}$, mechanical thrombectomy.

Supplementary Table 4. Univariable analysis of sICH (ECASS II) predictors

\begin{tabular}{|c|c|c|c|}
\hline Variable & $\mathrm{sICH}(\mathrm{n}=31)$ & No sICH $(n=130)$ & $P$ \\
\hline Age (yr) & $72.89 \pm 12.15$ & $67.74 \pm 15.98$ & 0.051 \\
\hline Female sex & $9(29.0)$ & $50(38.5)$ & 0.409 \\
\hline Dyslipidemia & $12(38.7)$ & $51(39.2)$ & 1.000 \\
\hline Diabetes mellitus & $9(29.0)$ & 14 (10.8) & 0.002 \\
\hline Hypertension & 22 (71.0) & $67(51.5)$ & 0.068 \\
\hline NIHSS & $18.53 \pm 3.46$ & $18.6 \pm 4.67$ & 0.925 \\
\hline Tobacco use & $6(19.4)$ & $45(34.6)$ & 0.133 \\
\hline ICA occlusion & $7(22.6)$ & $22(16.9)$ & 0.444 \\
\hline Delay till imaging (min) & $149.71 \pm 113.66$ & $163.7 \pm 131.43$ & 0.548 \\
\hline Drip and ship & $7(22.6)$ & 24 (18.5) & 0.609 \\
\hline iv tPA & $19(61.3)$ & $78(60.0)$ & 1.000 \\
\hline Volume core (mL) & $112.43 \pm 38.47$ & $100.6 \pm 35.67$ & 0.126 \\
\hline Volume T max >6 sec (mL) & $188.75 \pm 59.87$ & $171.58 \pm 72.5$ & 0.175 \\
\hline Mismatch ratio & $1.8 \pm 0.72$ & $1.76 \pm 0.7$ & 0.795 \\
\hline MT ( $P$ for vs. control) & $26(20.6)$ & $100(76.4)$ & 0.475 \\
\hline Control & $5(14.3)$ & 30 (85.7) & \\
\hline
\end{tabular}

Values are presented as number (\%) or mean \pm standard deviation.

sICH, symptomatic intracerebral hemorrhage; ECASS, European Cooperative Acute Stroke Study; NIHSS, National Institute of Health Stroke Scale; ICA, internal carotid artery; iv tPA, intravenous tissue plasminogen activator; MT, mechanical thrombectomy. 
Supplementary Table 5. Multivariable model of symptomatic intra-cranial hemorrhage predictors (pre specified adjustment for core volume, mismatch ratio and treatment group)

\begin{tabular}{llll}
\hline Variable & aOR & $95 \% \mathrm{Cl}$ & \multicolumn{1}{c}{ P } \\
\hline Age (yr) & 1.03 & $1.00-1.07$ & 0.051 \\
Hypertension & 1.15 & $0.43-3.03$ & 0.77 \\
Diabetes & 3.58 & $1.21-10.64$ & 0.02 \\
Volume core (each 10 mL) & 1.01 & $1.00-1.03$ & 0.02 \\
Core perfusion mismatch & 1.29 & $0.68-2.46$ & 0.43 \\
MT vs. Control & 1.94 & $0.59-6.26$ & 0.26 \\
\hline
\end{tabular}

$\mathrm{aOR}$, adjusted odds ratio; $\mathrm{Cl}$, confidence interval; $\mathrm{MT}$, mechanical thrombectomy. 


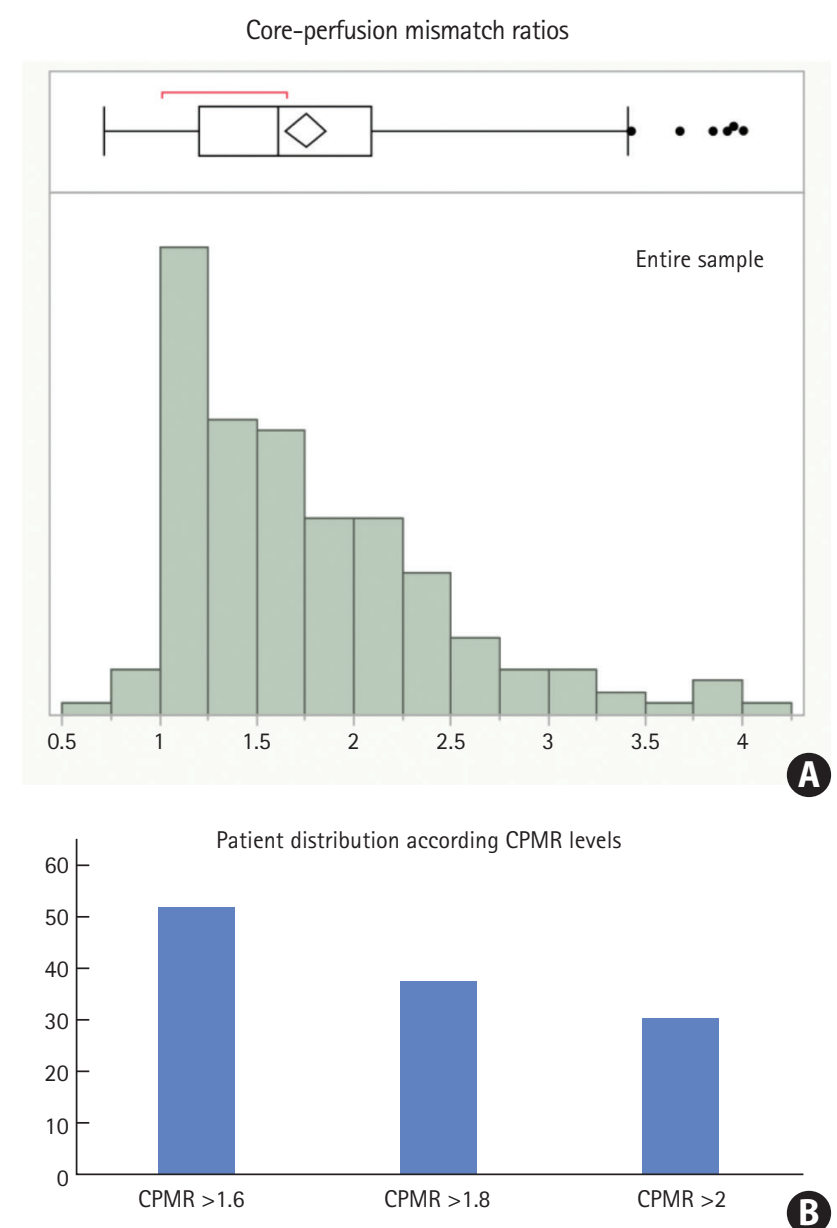

Supplementary Figure 1. Distribution of core perfusion mismatch ratios (CPMRs) in the entire sample (A) and proportion of patients with CPMRs above 1.6, 1.8, and 2 (B). 